\title{
A Fast Detection Algorithm for the X-Ray Pulsar Signal
}

\author{
Hao Liang and Yafeng Zhan \\ School of Aerospace Engineering, Tsinghua University, Beijing, China \\ Correspondence should be addressed to Yafeng Zhan; zhanyf@tsinghua.edu.cn
}

Received 22 August 2016; Revised 27 November 2016; Accepted 22 December 2016; Published 4 January 2017

Academic Editor: Yakov Strelniker

Copyright (C) 2017 H. Liang and Y. Zhan. This is an open access article distributed under the Creative Commons Attribution License, which permits unrestricted use, distribution, and reproduction in any medium, provided the original work is properly cited.

The detection of the X-ray pulsar signal is important for the autonomous navigation system using X-ray pulsars. In the condition of short observation time and limited number of photons for detection, the noise does not obey the Gaussian distribution. This fact has been little considered extant. In this paper, the model of the X-ray pulsar signal is rebuilt as the nonhomogeneous Poisson distribution and, in the condition of a fixed false alarm rate, a fast detection algorithm based on maximizing the detection probability is proposed. Simulation results show the effectiveness of the proposed detection algorithm.

\section{Introduction}

$\mathrm{X}$-ray pulsars are rapidly rotating neutron stars which could emit X-ray signals periodically, stably, and uniquely [1]. Since Downs first proposed interplanetary navigation using pulsars in 1974 [2], X-ray Pulsar Navigation (XPNAV) system has been considered in lots of scenarios for autonomous navigation [3-7]. XPNAV gets the spacecraft's position vector from measuring Time Difference of Arrival (TDOA) of the Xray pulsar signals between the spacecraft and the Solar System Barycenter (SSB). Because of the large distance (thousands of light years) between the X-ray pulsars and the solar system, the received X-ray pulsar signal is always weak and easy to be disturbed by kinds of noise [8]. It is difficult to recover the $\mathrm{X}$-ray pulsar profile [9] and get high precision TDOA, and each observation time for the X-ray pulsars may be as long as hundreds of seconds $[3,10]$. However, before using the $\mathrm{X}$ ray pulsar signals for navigation, we should firstly determine whether we have received the desired X-ray pulsar signals, which would help the detector's altitude controller to confirm the right direction of the X-ray pulsar. Unlike measuring TDOA, the signal detection process should be both accurate and fast. Therefore, it is a challenge to detect the weak X-ray pulsar signal with short time and limited photons.

The first widely used X-ray pulsar signal detection algorithm is based on the Fast Fourier Transform (FFT), which has been proved to be invalid when the signal's profile is nonsinusoidal or the noise is nongaussian [11]. The
Gregory-Loredo method parameterizes the number of bins for epoch folding and calculates the average ratio of the probability for the periodic model to the constant model [12]. The Gregory-Loredo method is effective on discovering new pulsars when there is no prior knowledge about the signal's shape; however, it does not use the prior information and is less efficient. The other detection algorithms, like $S$ transform [13,14] and Match Filter (MF) [15], consider the noise obeying the Gaussian distribution after epoch folding. However, that happens when the number of photons in the bins of epoch folding is larger than 20 [8]. For the detection problem, the observation time is not long enough to support approximating the Poisson noise by the Gaussian [16], and the algorithms above have this deficiency. Zhang et al. built the Poisson distribution model of X-ray pulsar and proposed a detection method by using the Bayesian theory [16]. However, it simply built the signal and noise by homogeneous Poisson distribution, which is imprecision. Besides that, it did not consider the influences of the unknown signal phase and the Doppler shift, which make it hard to be used in the practical scenario.

For XPNAV, the accurate prior knowledge about the Xray pulsar signal is essential. The prior knowledge includes the X-ray background noise's rate, the signal's flux, period, and profile. By using the prior information, we may build the Probability Density Function (PDF) [10] and get a fast detection algorithm based on Neyman-Pearson (NP) criterion [17]. Even in the condition of few photons for detection, 
the proposed algorithm's performance could meet detection requirements of XPNAV.

The rest of this paper is organized as follows. Section 2 introduces the X-ray pulsar signal model. Section 3 describes the fast detection algorithm in detail. Computer simulations are shown in Section 4 and some conclusions are given in the final part.

\section{X-Ray Pulsar Signal Model}

Unlike the radio signal, the $\mathrm{X}$-ray pulsar signal shows the particle property, which means the output of X-ray detector on the spacecraft is not an analog signal but a discrete arriving time sequence of individual photons. Assuming the observation time is $T_{\text {obs }}$, the number of received photons is $N_{T_{\text {obs }}}$, and the PDF of $N_{T_{\mathrm{obs}}}$ can be described by nonhomogeneous Poisson process [10]:

$$
P\left(N_{T_{\mathrm{obs}}}=k\right)=\frac{\left(\int_{0}^{T_{\mathrm{obs}}} \lambda(t) d t\right)^{k} \exp \left(-\int_{0}^{T_{\mathrm{obs}}} \lambda(t) d t\right)}{k !},
$$

where $\lambda(t)$ is the arriving rate of the photons as

$$
\lambda(t)=\lambda_{b}+\lambda_{s} h(\varphi(t)) .
$$

Here $\lambda_{b}$ is the X-ray background noise's rate which is timeinvariant in the cosmos environment, $\lambda_{s}$ is the X-ray pulsar signal's flux, $h(\varphi(t))$ is the X-ray pulsar signal's normalization standard profile template, $\varphi(t)=f_{r} \times t+\phi_{0}$ is the X-ray pulsar signal's phase, $f_{r}$ is the received frequency, and $\phi_{0}$ is the initial phase. Since the X-ray pulsar signal is periodic, $h(\varphi(t)+T)=$ $h(\varphi(t))$, where $T$ is the period.

\section{The Fast Detection Algorithm}

3.1. The Optimal NP Algorithm. We divide the observation timeline into $N$ equal bins, which is shown in Figure 1. The duration time of each of the bins is $\Delta t$. When $\Delta t$ is small enough, the arriving rate in the $n$th bin $\lambda_{n}(\boldsymbol{\theta})$ could be treated as a determined variable:

$$
\lambda_{n}(\boldsymbol{\theta}) \approx \frac{1}{\Delta t} \int_{t_{0}+(n-1) \Delta t}^{t_{0}+n \Delta t} \lambda(t ; \boldsymbol{\theta}) d t
$$

where $\boldsymbol{\theta}=\left[\begin{array}{ll}f_{r} & \phi_{0}\end{array}\right]^{\mathrm{T}}$; the supper index T represents the transpose of a vector. The number of photons in $n$th bin $x_{n}$ is a random variable with the PDF:

$$
p\left(x_{n}=k ; \boldsymbol{\theta}\right)=\frac{\left(\lambda_{n}(\boldsymbol{\theta}) \Delta t\right)^{k}}{k !} \exp \left(-\lambda_{n}(\boldsymbol{\theta}) \Delta t\right) .
$$

Since the random variables in the different bins are independent, the Joint Probability Density Function (JPDF) could be expressed as

$$
\begin{aligned}
p(\mathbf{X} ; \boldsymbol{\theta}) & =\prod_{n=1}^{N} p\left(x_{n} ; \boldsymbol{\theta}\right) \\
& =\prod_{n=1}^{N} \frac{\left(\lambda_{n}(\boldsymbol{\theta}) \Delta t\right)^{x_{n}}}{x_{n} !} \exp \left(-\lambda_{n}(\boldsymbol{\theta}) \Delta t\right),
\end{aligned}
$$

where $\mathbf{X}=\left(x_{1}, x_{2}, \ldots, x_{N}\right)$.

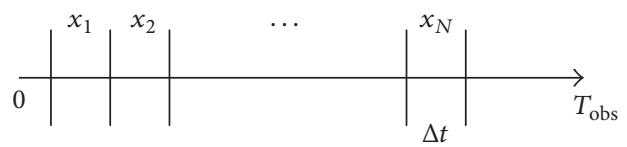

FIGURE 1: The observation timeline.

If the X-ray pulsar signal exists in the received photons, $\lambda_{n}(\boldsymbol{\theta})=\lambda_{b}+\lambda_{s} h(\boldsymbol{\theta})$, then the JPDF of $\mathbf{X}$ could be expressed as

$$
\begin{aligned}
& H 1: p_{1}(\mathbf{X} ; \boldsymbol{\theta}) \\
& =\prod_{n=1}^{N} \frac{\left(\left(\lambda_{b}+\lambda_{s} h(\boldsymbol{\theta})\right) \Delta t\right)^{x_{n}}}{x_{n} !} \exp \left(-\left(\lambda_{b}+\lambda_{s} h(\boldsymbol{\theta})\right) \Delta t\right) .
\end{aligned}
$$

If the X-ray pulsar signal does not exist in the received photons, $\lambda_{n}(\boldsymbol{\theta})=\lambda_{b}$, then the JPDF of $\mathbf{X}$ could be expressed as

$$
\text { H0: } p_{0}(\mathbf{X} ; \boldsymbol{\theta})=\prod_{n=1}^{N} \frac{\left(\lambda_{b} \Delta t\right)^{x_{n}}}{x_{n} !} \exp \left(-\lambda_{b} \Delta t\right) .
$$

In the condition of a fixed probability of false alarm $p_{\mathrm{FA}}$, NP criterion maximizes the detection probability [17]. Based on NP criterion, we could do the Likelihood Ratio Test (LRT):

$$
L(\mathbf{X} ; \boldsymbol{\theta})=\frac{p_{1}(\mathbf{X} ; \boldsymbol{\theta})}{p_{0}(\mathbf{X} ; \boldsymbol{\theta})}=\gamma .
$$

If $\gamma$ is larger than the threshold $\gamma_{\mathrm{th}}$, we can determine that we have received the X-ray pulsar signal. Otherwise, the X-ray pulsar signal does not exist in the received photons. Equation (8) could be simplified as follows:

$$
\begin{aligned}
& L(\mathbf{X} ; \boldsymbol{\theta})=\frac{p_{1}(\mathbf{X} ; \boldsymbol{\theta})}{p_{0}(\mathbf{X} ; \boldsymbol{\theta})} \\
& =\prod_{n=1}^{N}\left(\frac{\lambda_{n}(\boldsymbol{\theta})}{\lambda_{b}}\right)^{x_{n}} \exp \left(\left(\lambda_{b}-\lambda_{n}(\boldsymbol{\theta})\right) \Delta t\right), \\
& \ln (L(\mathbf{X} ; \boldsymbol{\theta})) \\
& =\sum_{n=1}^{N} \ln \left(\frac{\lambda_{n}(\boldsymbol{\theta})}{\lambda_{b}}\right)^{x_{n}}+\sum_{n=1}^{N}\left(\lambda_{b}-\lambda_{n}(\boldsymbol{\theta})\right) \Delta t, \\
& \ln (L(\mathbf{X} ; \boldsymbol{\theta}))+\sum_{n=1}^{N}\left(\lambda_{s}(\boldsymbol{\theta})\right) \Delta t \\
& =\sum_{n=1}^{N} x_{n}\left(\ln \left(\lambda_{n}(\boldsymbol{\theta})\right)-\ln \left(\lambda_{b}\right)\right) \text {. }
\end{aligned}
$$

Since $\sum_{n=1}^{N}\left(\lambda_{s}(\boldsymbol{\theta})\right) \Delta t$ is independent of the measurement variable $x_{n}$, the left side of (11) could be added to the threshold, and then

$$
\sum_{n=1}^{N} x_{n}\left(\ln \left(\lambda_{n}(\boldsymbol{\theta})\right)-\ln \left(\lambda_{b}\right)\right)=\gamma^{\prime}
$$


TABLE 1: The parameters of X-ray pulsar sources.

\begin{tabular}{|c|c|c|c|c|c|}
\hline ID & Name & RA(J2000) (hh:mm:ss) & DE(J2000) (dd:mm:ss) & Flux (photons $/ \mathrm{cm}^{2} / \mathrm{s}$ ) & Period (s) \\
\hline 1 & B0540-69 & $05: 39: 39$ & $-69: 44: 36$ & $5.15 e \times 10^{-3}$ & 0.0505 \\
\hline 2 & B1744-24A & $17: 48: 02$ & $-24: 46: 38$ & $1.09 \times 10^{-3}$ & 0.0116 \\
\hline 3 & B1823-13 & $18: 26: 13$ & $-13: 34: 47$ & $2.63 \times 10^{-3}$ & 0.1015 \\
\hline
\end{tabular}

If $\Delta t \rightarrow 0$, there is only one or none photon in each bin, and then (12) could be converted as

$$
G(\varphi)=\sum_{i=1}^{M}\left(\ln \left(\lambda\left(\varphi_{i}\right)\right)-\ln \left(\lambda_{b}\right)\right)=\gamma^{\prime},
$$

where $M$ is the total number of the photons, $\varphi=$ $\left[\begin{array}{llll}\varphi_{1} & \varphi_{2} & \cdots & \varphi_{M}\end{array}\right]^{\mathrm{T}}, \varphi_{i}$ is the phase of $i$ th photons, which could be calculated by

$$
\varphi_{i}=f_{r} \cdot t_{i}+\phi_{0} .
$$

From (13), the optimal detection variable is $G(\varphi)$. Thus, the steps of the optimal NP detection algorithm for the X-ray pulsar signal are as below:

(I) The X-ray detector on-board receives the photons and notes each photons' arriving time $t_{i}$.

(II) Convert the transferred arriving time $t_{i}$ into $\varphi_{i}$ using (14).

(III) Search the arriving rate $\lambda\left(\varphi_{i}\right)$ of each arriving time from the XPNAV database (since $\lambda\left(\varphi_{i}\right)$ is periodic, the XPNAV database is a table which stores one period of the function $\lambda\left(\varphi_{i}\right)$ ), and calculate the logarithm $\ln \left(\lambda\left(\varphi_{i}\right)\right)$.

(IV) Add up all $\ln \left(\lambda\left(\varphi_{i}\right)\right)$, and compare the summation $G(\varphi)$ with $\gamma_{\text {th }}$.

The prior knowledge before the detection process includes the XPNAV database about the arriving rate functions $\lambda(t)$ and $\gamma_{\text {th }}$. The XPNAV database could be built by observing the X-ray pulsars for months and some explorers have tried to do that, such as the Rossi X-Ray Timing Explorer (RXTE) from the National Aeronautics and Space Administration (NASA) and the Röntgen Satellite (ROSAT) from the German Aerospace Center (DLR). It is difficult to get an analytical expression for $\gamma_{\text {th }}$. Monte Carlo simulations would be used to calculate $\gamma_{\text {th }}$. The calculation process is as below:

(I) Set a fixed probability of false alarm $p_{\mathrm{FA}}$.

(II) Generate the arriving time of the photons based on the Poisson distribution with the arriving rate $\lambda_{b}$.

(III) Calculate $G(\varphi)$ based on the above steps of the optimal NP detection algorithm.

(IV) Repeat step (II) and step (III) for $K$ times; $K$ is the times of the Monte Carlo simulations.

(V) Rank each simulation result in descending order, and set a threshold $\gamma_{\text {th }}$ to make the proportion of $G(\varphi)$ greater than $\gamma_{\text {th }}$ equal $p_{\mathrm{FA}} \cdot \gamma_{\text {th }}$ is the threshold we pursue.
3.2. The Suboptimal NP Algorithm. Besides the prior knowledge, how to convert the arriving time $t_{i}$ into the phase $\varphi_{i}$ is another important issue. $\phi_{0}$ is different due to the spacecraft's position, and $f_{r}$ could be affected by the Doppler shift due to the spacecraft's velocity:

$$
\begin{aligned}
\varphi_{i} & =f_{r} \times t_{i}+\phi_{0}=\left(f_{0}+f_{d}\right) \times t_{i}+\phi_{0} \\
f_{d} & =\frac{\mathbf{v} \cdot \mathbf{r}}{c} f_{0}
\end{aligned}
$$

where $f_{0}$ is the fixed frequency of the X-ray pulsar, $f_{d}$ is the Doppler frequency, $\mathbf{v}$ is the spacecraft's velocity vector, $\mathbf{r}$ is the X-ray pulsar's direction vector, and $c$ is the light velocity. Supposing there are no prior distributions of $\phi_{0}$ and $f_{d}$, we could use the two-dimension search based on the Maximum Likelihood (ML) estimation [10] to deal with the issue.

Assuming $\phi_{0}$ 's searching range is $[0,1)$, dividing the range into $p$ segments, the $j$ th searching phase is $\phi_{0}^{j}$. Dividing the $f_{d}$ 's searching range into $q$ segments, the $k$ th searching frequency is $f_{d}^{k}$. Then, take $\phi_{0}^{j}$ and $f_{d}^{k}$ into step (II) of the optimal NP detection algorithm and calculate $G_{j, k}\left(\varphi ; \phi_{0}^{j}, f_{d}^{k}\right)$. $\phi_{0}$ and $f_{d}$ in $\max \left(G_{j, k}\left(\varphi ; \phi_{0}^{j}, f_{d}^{k}\right)\right)$ have the maximum probability equaling the real ones, where $\max (\cdot)$ is the maximum function. Therefore, we could use $\max \left(G_{j, k}\left(\varphi ; \phi_{0}^{j}, f_{d}^{k}\right)\right)$ as the detection variable to be compared with $\gamma_{\text {th }}$. We may call the algorithm resolving the unknown $\phi_{0}$ and $f_{d}$ as the suboptimal NP detection algorithm.

For the practical navigation application, $\phi_{0}$ and $f_{d}$ are always unknown, so the suboptimal NP detection algorithm is more useful than the optimal one.

\section{Computer Simulations}

Three weak X-ray pulsars (B0540-69, B1744-24A, and B182313) are selected to simulate the proposed detection algorithm's performance. The parameters of the X-ray pulsars are shown in Table 1, and the normalization standard profile templates [18] are shown in Figure 2. The X-ray background noise's rate $\lambda_{b}$ is 0.005 photons $/ \mathrm{s} / \mathrm{cm}^{2}$. The X-ray pulsar signal's flux $\lambda_{s}$ could be obtained from Table 1 [1]. The X-ray detector's area is $1 \mathrm{~m}^{2}$, and the fixed probability of false alarm $p_{\mathrm{FA}}$ is set as 0.001 .

The initial phase $\phi_{0}$ obeys the uniform distribution with range $[0,1)$. The unknown velocity $v$ obeys the uniform distribution with range $(-7.9,7.9) \mathrm{km} / \mathrm{s}$, in which $7.9 \mathrm{~km} / \mathrm{s}$ is the first cosmic velocity. The Doppler frequency $f_{d}$ could be calculated by (16). The method to generate the photons refers to reference [10].

The MF algorithm calculates the correlation peak for the X-ray pulsar signal's profile with the standard signal 

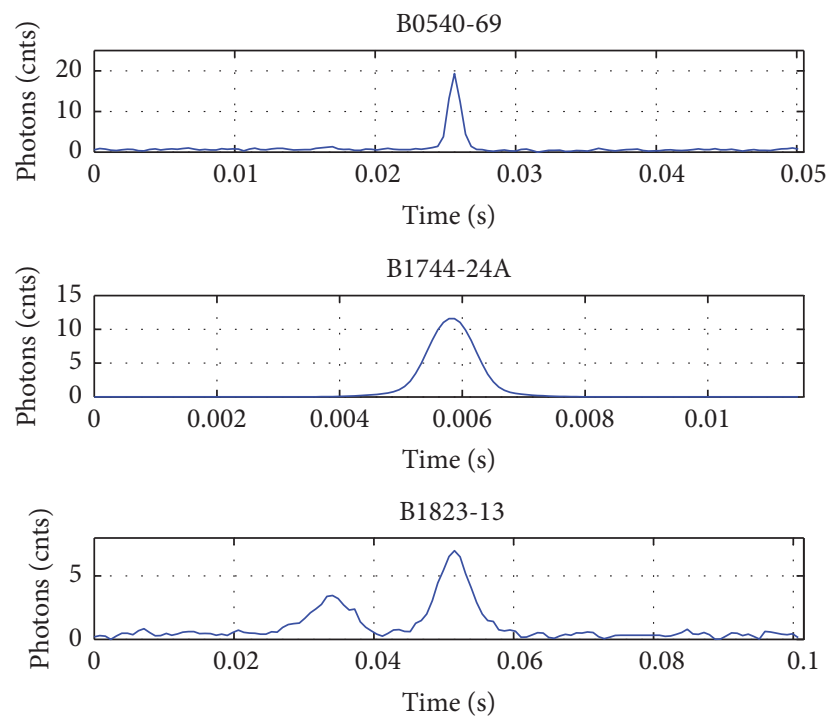

FIGURE 2: The normalization standard profile templates of three Xray pulsars.

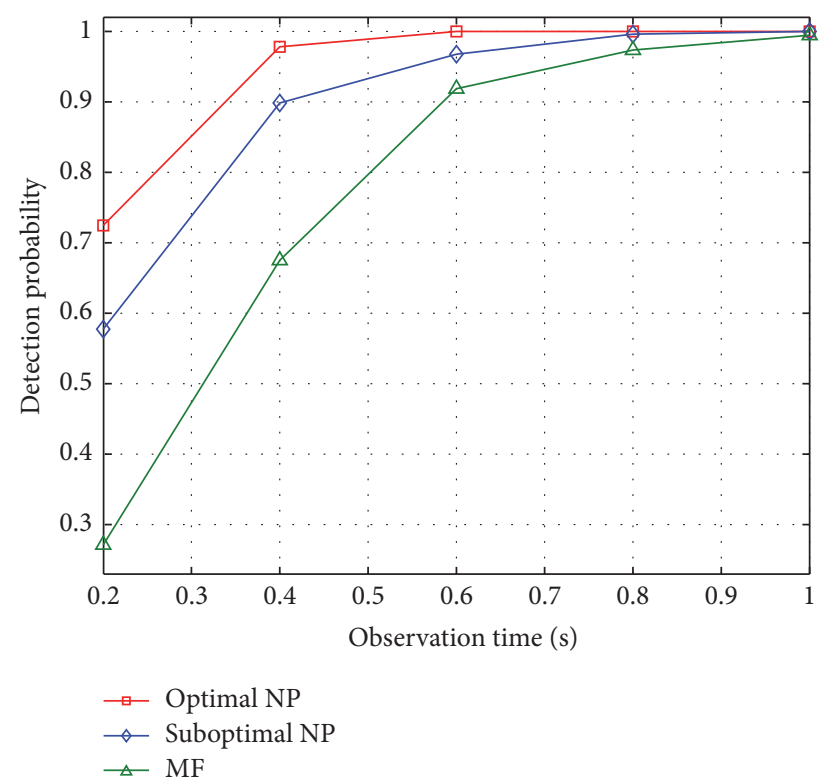

FIGURE 3: The detection probability for B0540-69.

template [15]. Since the MF algorithm is optimal when the noise is obeying the Gaussian distribution, we choose the MF algorithm for comparison.

We divide the simulation algorithm into three groups: (I) the optimal NP detection algorithm; (II) the suboptimal NP detection algorithm, and the number of segments for searching both $\phi_{0}$ and $f_{d}$ is set as 10; (III) the MF algorithm with unknown information about $\phi_{0}$ and $f_{d}$, and the number of bins for epoch folding is 64 . We perform the Monte Carlo simulations for 1000 times to calculate the detection probability, and the results are shown from Figures 3-5.

From Figures 3-5, the detection probability increases with the increasing observation time. Meanwhile, in the

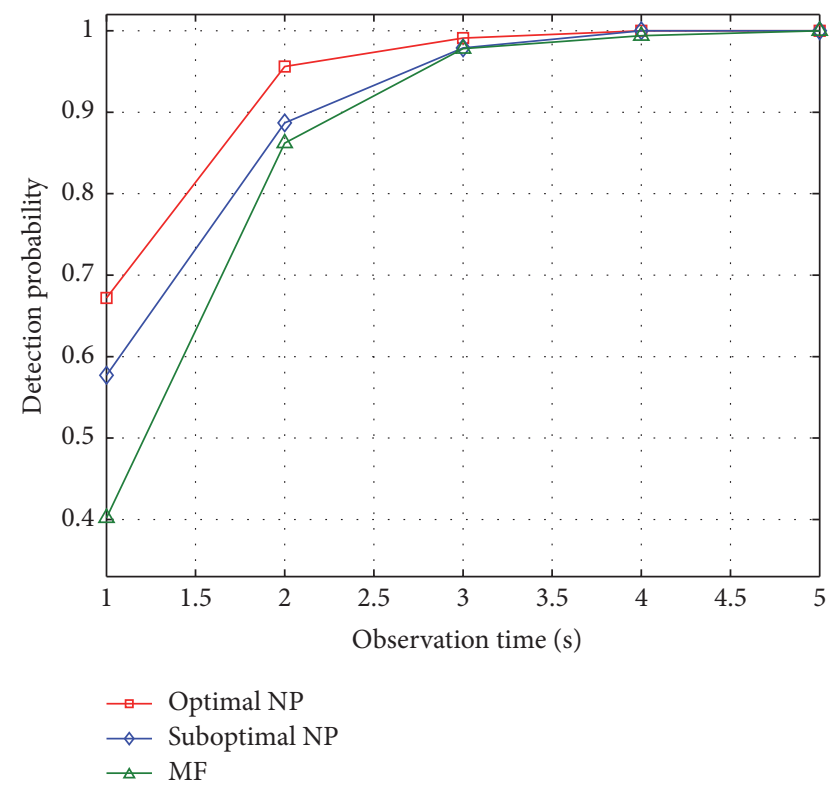

FIGURE 4: The detection probability for B1744-24A.

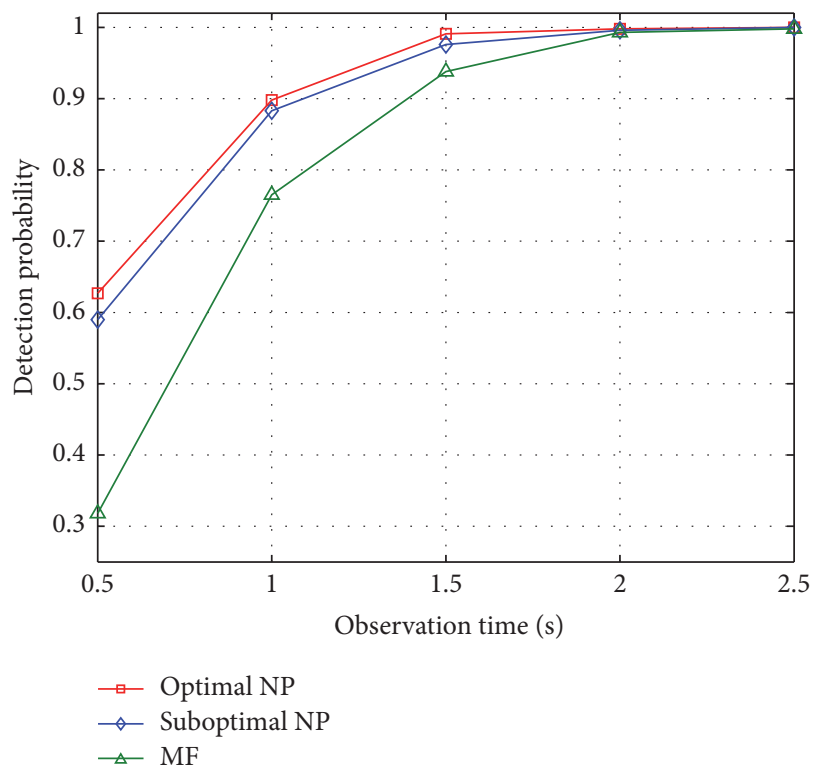

FIGURE 5: The detection probability for B1823-13.

same simulation conditions, the suboptimal NP detection algorithm's detection probability is better than the MF's. The MF algorithm is effective when the noise is obeying the Gaussian distribution. However, in the condition of short observation time and limited photons, the noise obeys Poisson distribution, which makes the MF algorithm less effective than the proposed detection algorithm.

Since the limited number of photons, the estimation for $\phi_{0}$ and $f_{d}$ based on ML may be imprecise, and the detection probability of the suboptimal NP detection algorithm is lower than the optimal NP detection algorithm. Therefore, the optimal NP detection algorithm is a performance bound. 


\section{Conclusion}

The detection for the X-ray pulsar signal is important for XPNAV and some other navigation scenarios using X-ray pulsars. In this paper, we propose a fast detection algorithm for the X-ray pulsar signal based on NP criteria. Computer simulations show that, in the condition of short observation time and limited number of photons, the detection probability of the proposed algorithm is still high. Besides that, the fast detection algorithm may be extended to other detection issues when the signals obey the Poisson distribution.

\section{Competing Interests}

The authors declare that they have no competing interests.

\section{Acknowledgments}

This work was supported by National Natural Science Foundation of China (61271265 and 61671263) and Tsinghua University Initiative Scientific Research Program (2013089244 and 20161080057).

\section{References}

[1] S. I. Sheikh, The use of variable celestial X-ray sources for spacecraft navigation [Ph.D. thesis], University of Maryland, 2005.

[2] G. S. Downs, Interplanetary Navigation Using Pulsating Radio Sources, NASA, 1974.

[3] S. I. Sheikh, D. J. Pines, P. S. Ray, K. S. Wood, M. N. Lovellette, and M. T. Wolff, "Spacecraft navigation using X-ray pulsars," Journal of Guidance, Control, and Dynamics, vol. 29, no. 1, pp. 49-63, 2006.

[4] A. A. Emadzadeh and J. L. Speyer, "Relative navigation between two spacecraft using X-ray pulsars," IEEE Transactions on Control Systems Technology, vol. 19, no. 5, pp. 1021-1035, 2011.

[5] E. Wei, S. Jin, Q. Zhang, J. Liu, X. Li, and W. Yan, "Autonomous navigation of Mars probe using X-ray pulsars: modeling and results," Advances in Space Research, vol. 51, no. 5, pp. 849-857, 2013.

[6] X. Kai, W. Chunling, and L. Liangdong, "The use of X-ray pulsars for aiding navigation of satellites in constellations," Acta Astronautica, vol. 64, no. 4, pp. 427-436, 2009.

[7] Y. Zhou, P. Wu, and X. Li, "Autonomous navigation for lunar satellite using X-ray pulsars with measurement faults," IET Science, Measurement \& Technology, vol. 10, no. 3, pp. 239-246, 2016.

[8] J. Hanson, S. Sheikh, P. Graven, and J. Collins, "Noise analysis for X-ray navigation systems," in Proceedings of the IEEE/ION Position, Location and Navigation Symposium (PLANS '08), pp. 704-713, Monterey, Calif, USA, May 2008.

[9] D. Zhang, W. Zheng, Y. Wang, and L. Zhang, "X-ray pulsar profile recovery based on tracking-differentiator," Mathematical Problems in Engineering, vol. 2016, Article ID 4238165, 10 pages, 2016.

[10] A. A. Emadzadeh and J. L. Speyer, "On modeling and pulse phase estimation of X-ray pulsars," IEEE Transactions on Signal Processing, vol. 58, no. 9, pp. 4484-4495, 2010.

[11] P. C. Gregory and T. J. Loredo, "Bayesian periodic signal detection: analysis of ROSAT observations of PSR 0540-693," The Astrophysical Journal, vol. 473, no. 2, pp. 1059-1066, 1996.
[12] P. C. Gregory and T. J. Loredo, "A new method for the detection of a periodic signal of unknown shape and period," The Astrophysical Journal, vol. 398, no. 1, pp. 146-168, 1992.

[13] L. Wang, L.-P. Xu, H. Zhang, and N. Luo, "Pulsar signal detection based on S-transform," Acta Physica Sinica, vol. 62, no. 13, pp. 596-605, 2013.

[14] L. Wang, L.-P. Xu, and H. Zhang, "A constant false alarm rate detection algorithm for X-ray pulsar signal based on Stransform," Journal of Astronautics, vol. 35, no. 8, pp. 931-937, 2014.

[15] C. Kabakchiev, V. Behar, P. Buist et al., "Detection and estimation of pulsar signals for navigation," in Proceedings of the IEEE 16th International Radar Symposium, pp. 688-693, Dresden, Germany, June 2015.

[16] H. Zhang, L.-P. Xu, Q. Xie, and N. Luo, "X-ray pulsar weak signal detection based on Bayesian estimation," Acta Physica Sinica, vol. 60, no. 4, pp. 829-835, 2011.

[17] S. M. Key, Fundamentals of Statistical Signal Processing, Vol. II, Detection Theory, Prentice Hall, 1998.

[18] HEASARC (High Energy Astrophysics Science Archive Research Center) Browse: Main Interface, http://heasarc.gsfc .nasa.gov/cgi-bin/W3Browse/w3browse.pl. 


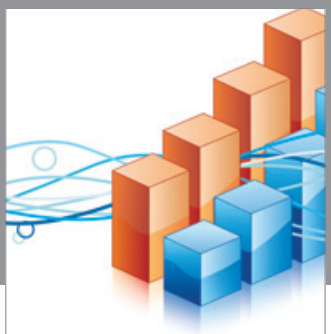

Advances in

Operations Research

vatem alat4

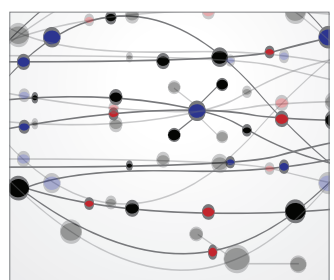

\section{The Scientific} World Journal
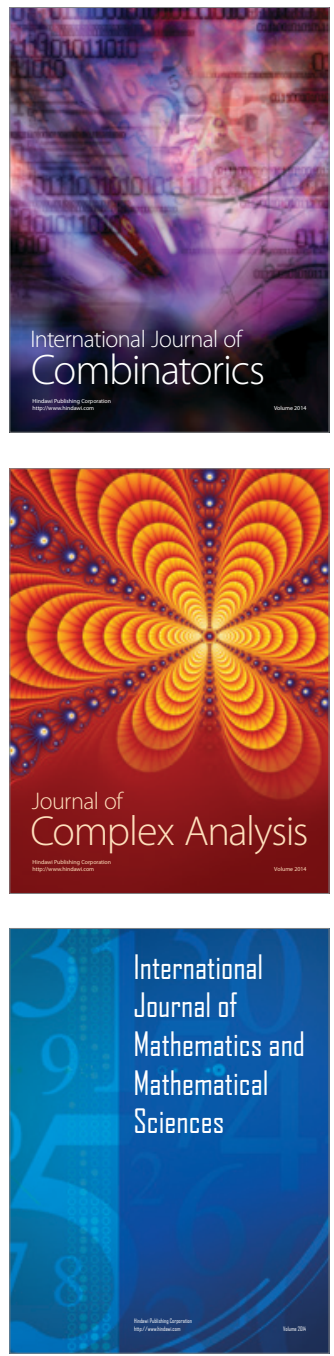
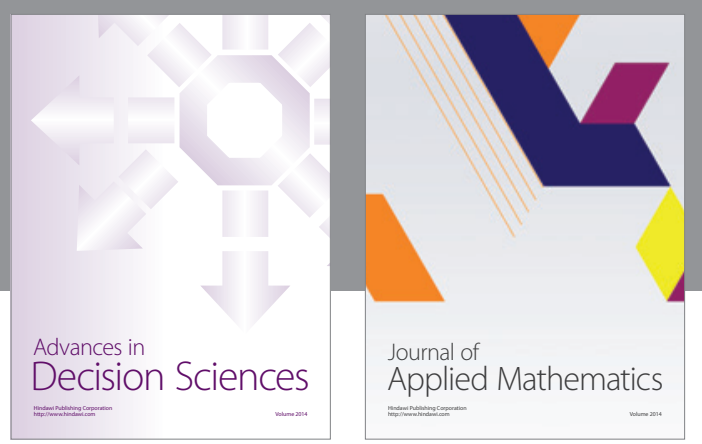

Algebra

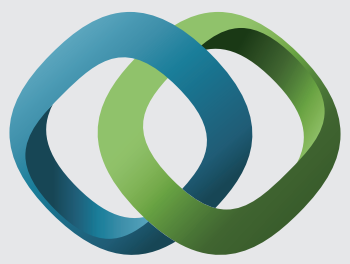

\section{Hindawi}

Submit your manuscripts at

https://www.hindawi.com
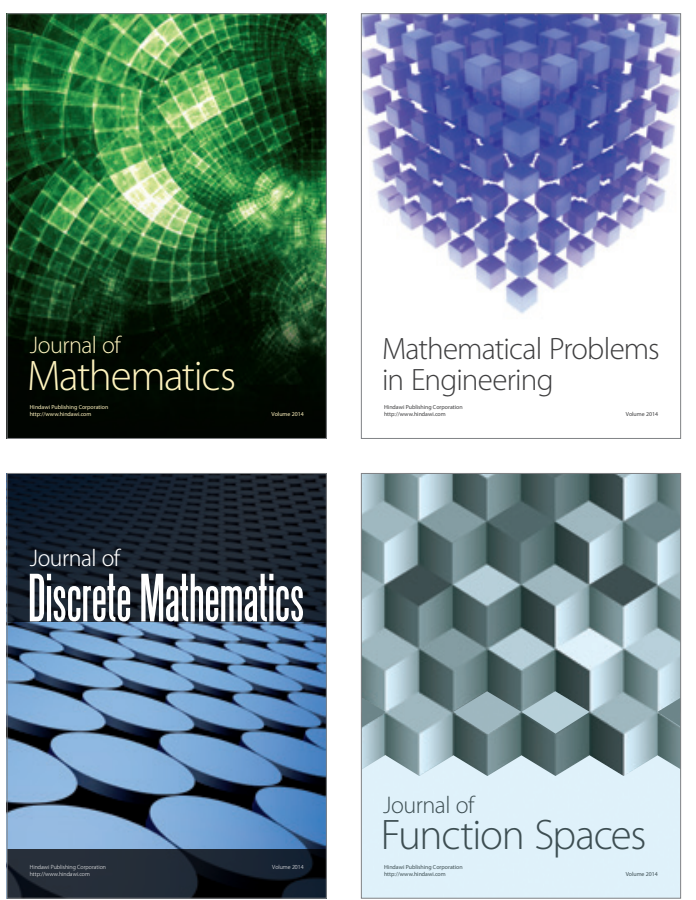

Mathematical Problems in Engineering
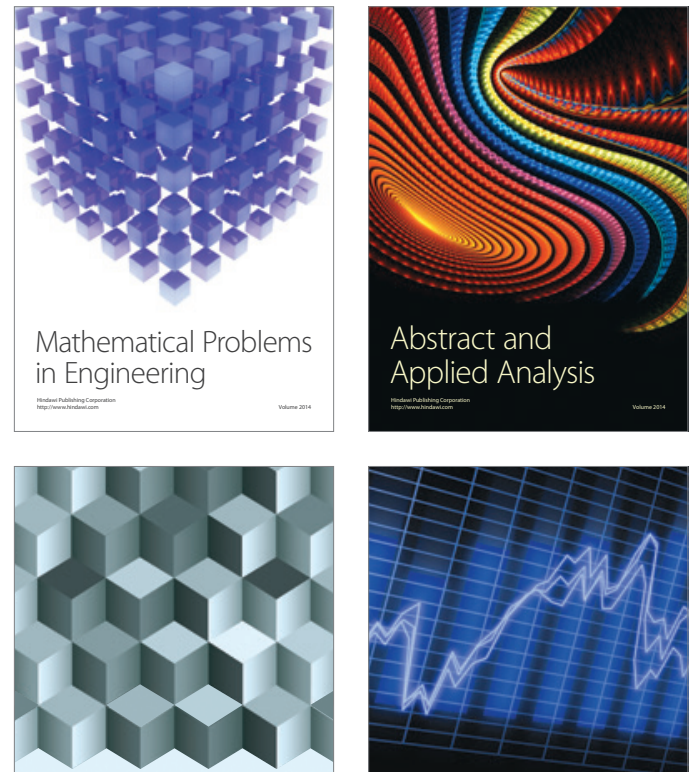

Journal of

Function Spaces

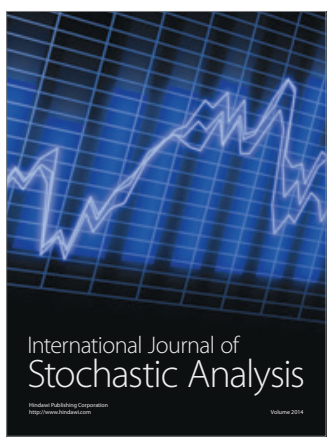

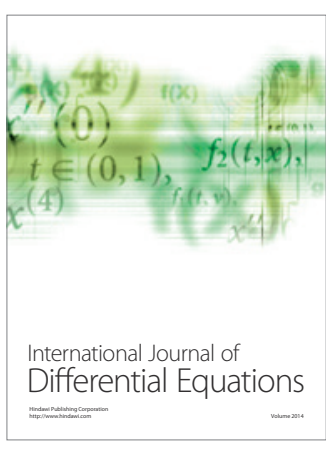
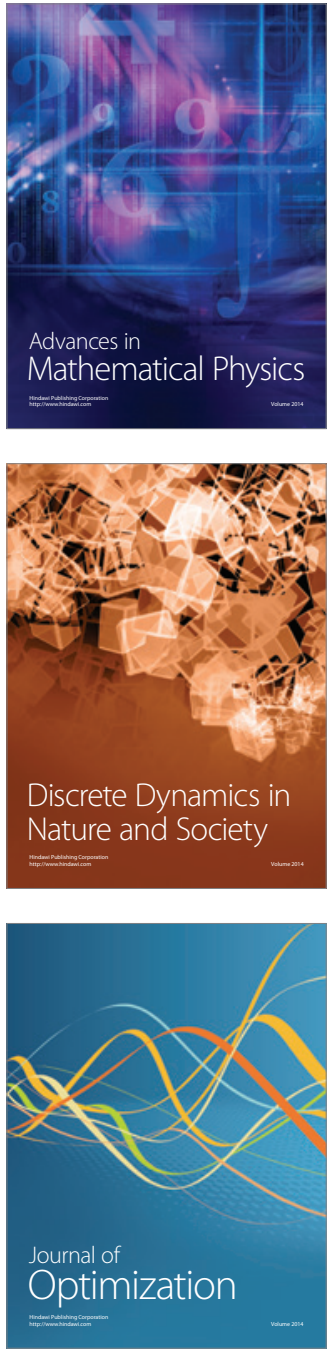